\title{
Motor Unit Regulation of Mammalian Pharyngeal Dilator Muscle Activity
}

Erik van Lunteren and Thomas E. Dick

Pulmonary Division, Department of Medicine, Case Western Reserve University, and University Hospitals of Cleveland, Cleveland, Ohio 44106

\begin{abstract}
The present study examined the cellular regulation of one of the pharyngeal dilator muscles, the geniohyoid, by assessing its motor unit (MU) behavior in anesthetized cats. During spontaneous breathing, MU that (a) were active during inspiration only (I-MU) and (b) were active during both inspiration and expiration (I/E-MU) were identified. I-MU had a later inspiratory onset time and a shorter duration of inspiratory firing than did I/E-MU $(P<0.002$ and $P<0.0001$, respectively). I-MU were usually quiescent whereas I/E-MU were usually active during the last $20 \%$ of inspiration. I/E-MU fired more rapidly $(P<0.00001)$ and for relatively longer periods of time $(P<0.00001)$ during inspiration than during expiration. End-expiratory airway occlusion (preventing lung expansion during inspiration) augmented the inspiratory activity of both I-MU and I/E-MU. Conversely, end-expiratory airway occlusion reduced the absolute and relative firing durations ( $P$ $<0.002$ and $P<0.00002$, respectively) and the firing frequency $(P<0.001)$ of I/E-MU activity during expiration. These results indicate that $(a)$ the complex pattern of pharyngeal dilator muscle activity is due to the integrated activity of a heterogeneous group of $\mathrm{MU},(b)$ changes in the degree to which pharyngeal dilator muscles are active result from combinations of MU recruitment/decruitment and modulations of the frequency and duration of MU firing, and (c) gating of lung-volume afferent information occurs during the respiratory cycle.
\end{abstract}

\section{Introduction}

Upper airway patency may be impaired under a variety of circumstances, including after administration of anesthetics and sedatives, as a result of a variety of neurological conditions leading to alterations in the level of consciousness, and during sleep (1-7). The resulting interference with ventilatory homeostasis leads to hypoxia and hypercapnia, with their attendant cardiovascular, neurologic, and other systemic consequences. The important roles that pharyngeal dilators, such as the genioglossus, geniohyoid and sternothyroid muscles, play in restoring and maintaining upper airway stability are well established. In both humans and in other mammals, activation of pharyngeal dilator muscles increases the ability of the pharynx

This work was presented in part at the 18th Annual Meeting of the Society for Neuroscience, Toronto, Ontario, Canada, 13-18 November 1988.

Received for publication 29 August 1988 and in revised form 3 March 1989.

J. Clin. Invest.

(C) The American Society for Clinical Investigation, Inc. 0021-9738/89/08/0577/09 \$2.00

Volume 84, August 1989, 577-585 to remain patent in the face of subatmospheric intraluminal pressures produced during inspiration (6-11). The electrical and mechanical activity of the pharyngeal dilator muscles is influenced by many of the same stimuli which affect breathing $(9,12-20)$, allowing the upper airway musculature to act synergistically with the thoracic respiratory muscles in responding to ventilatory perturbations.

A motor unit (MU) ${ }^{1}$ consists of a single motoneuron and the muscle fibers it supplies, and is the fundamental entity by which activation of a striated muscle is controlled (21). During graded contractions, the force output of limb skeletal muscles is augmented both by increasing the frequency of MU firing and by the recruitment of additional MU. For the diaphragm and phrenic nerve, subpopulations of MU exist, and the responses of these subpopulations to chemical and mechanical stimuli are well characterized (22-25). Although MU recordings from the hypoglossal nerve have been reported previously $(12,26,27)$, this nerve innervates multiple upper airway muscles with diverse mechanical actions: muscles that dilate the pharynx (the genioglossus muscle, which protrudes the tongue, and the geniohyoid muscle, which moves the hyoid arch anteriorly), muscles that narrow the pharynx (the styloglossus and hypoglossal muscles, which both retract the tongue), and muscles with as yet uncharacterized effects on upper airway patency (the intrinsic tongue muscles, which alter the shape of the tongue). This functionally mixed motor innervation precludes application of data on hypoglossal nerve activity towards a clear understanding of the cellular control of pharyngeal patency, and in addition, may contribute to discrepant views regarding the influences of pulmonary stretch receptor afferents on upper airway muscle activity during expiration $(12,28-32)$. In the present study we therefore recorded electrical activity from MU of a well-defined pharyngeal dilator, the geniohyoid muscle $(8,9,11,19,33-35)$, to (a) define $\mathrm{MU}$ firing patterns accounting for the complex pattern of electrical activity recorded from pharyngeal dilator muscles, $(b)$ delineate MU strategies by which alterations in muscle activation are produced, $(c)$ ascertain whether distinct subpopulations of MU can be identified, and $(d)$ test the effects of lung volumerelated afferents on MU activity during inspiration and especially during expiration.

\section{Methods}

The present studies were performed in 12 adult cats. All protocols were approved by the animal experimentation panel at Case Western Reserve University before the start of the studies, and all procedures followed the animal care guidelines issued by the National Institutes of

1. Abbreviations used in this paper: EMG, electromyogram; I-MU, motor unit that was active only during inspiration (of unoccluded breaths); I/E-MU, motor unit that was active during both inspiration and expiration (of unoccluded breaths); MU, motor unit(s); Te, expiratory time; $\mathrm{Ti}$, inspiratory time. 
Health and by the American Physiological Society. The animals were anesthetized with intraperitoneal pentobarbital sodium (initial dose of $35-40 \mathrm{mg} / \mathrm{kg}$ ) and placed supine on a heating blanket. The femoral artery and vein were catheterized to monitor blood pressure and to administer supplemental doses of anesthesia, respectively. Assessment of the level of anesthesia included monitoring respiratory rate, heart rate, pupillary diameter, and the corneal reflex, and verifying the absence of spontaneous nonrespiratory movements and of movement responses to tactile stimuli. The trachea was cannulated via a midline cervical incision and connected via a heated pneumotachograph (Fleisch No. 00, OEM Medical, Richmond, VA) to a low dead space $(1.6 \mathrm{ml})$ non-rebreathing respiratory valve (model 2384, Hans Rudolph, Kansas City, MO). The pressure drop across the pneumotachograph was measured using a differential pressure transducer $( \pm 2 \mathrm{~cm}$ $\mathrm{H}_{2} \mathrm{O}$, Validyne Engineering Corp., Northridge, CA) to record respiratory airflow, and this value was electronically integrated (FV156 Integrator, Validyne Engineering Corp.) to obtain traces of tidal volume.

Electromyographic (EMG) recordings from the diaphragm and geniohyoid muscles were obtained using bipolar electrodes fabricated from 0.005 -in diam Teflon-coated steel wire (A-M Systems, Inc., Everett, WA), with bare tips of $1 \mathrm{~mm}$ and tip separation of $4 \mathrm{~mm}(9$, $16-19,31$ ). Electrodes were inserted into the costal diaphragm through a small midline abdominal incision which was subsequently closed. The geniohyoid muscle was exposed via a midline ventral cervical incision by removing overlying tissue including part of the mylohyoid muscle, allowing visual verification of electrode placement. EMG recordings were amplified at band-pass filter settings of $30 \mathrm{~Hz}$ to $3 \mathrm{kHz}$ (P511 amplifier, Grass Instrument Co., Quincy, MA), rectified and passed through Paynter filters with a 200-ms time constant (MA-821S moving averager, Charles Ward Enterprises, Ardmore, PA) to obtain moving average EMGs $(9,16-19,31)$. Amplified and moving average EMGs were recorded simultaneously with tidal airflow and/or tidal volume on an oscillographic recorder (VR-12, Electronics for Medicine, Pleasantville, NY).

Electrical activity was recorded from single MU of the geniohyoid muscle using either the above conventional electrodes or bipolar tungsten electrodes. The latter electrodes were fabricated from two insulated 0.005 -in diam tungsten microelectrodes (A-M Systems, Inc.), whose tips were glued together so that their tapered ends were $<0.5$ $\mathrm{mm}$ apart $(25,36)$. Coiled fine wire connected each electrode to a set of amplifier leads, and electrical recordings were amplified (P511, Grass Instrument Co.), displayed on an oscilloscope (model 5513, Tektronix, Inc., Beaverton, OR) to monitor size and shape of MU impulses, recorded on the oscillographic recorder, and fed to an audio monitor. Single- and pauci-MU recordings were passed through a time-amplitude window discriminator (DIS-1, Bak Electronics, Rockville, MD), and the discriminated pulses were displayed on the oscilloscope and/or recorded on the oscillographic recorder.

Diaphragm, geniohyoid muscle, and geniohyoid MU electrical activities were recorded while the animals spontaneously breathed $7 \%$ $\mathrm{CO}_{2}$ in $\mathrm{O}_{2}$ or $100 \% \mathrm{O}_{2}$. In previous work we found that in anesthetized cats the geniohyoid muscle was rarely electrically active during resting oxygen breathing (19), and this was also the case in the present study. We therefore obtained most of the electrical recordings from geniohyoid MU while the animals inspired the hypercapnic gas mixture to ensure that $\mathrm{MU}$ with a broad range of $\mathrm{CO}_{2}$ thresholds were assessed. MU were distinguished in the same animal by contralateral location, different firing patterns of any type during simultaneous recording, and inspiratory versus inspiratory/expiratory firing patterns during nonsimultaneous recording while the animal inspired the same gas mixture. The recordings of MU activity were obtained both ipsilateral and contralateral to recordings of EMG from the whole muscle. After baseline MU firing patterns were recorded, the effects of lung volumerelated afferents were assessed by occluding the inspiratory port of the respiratory valve during expiration, thereby preventing lung expansion during the subsequent inspiration (end-expiratory occlusion), or by occluding the expiratory port of the respiratory valve during inspiration thereby maintaining lung expansion during the subsequent expi- ration (end-inspiratory occlusion). MU firing patterns were related to the inspiratory and expiratory phases of the respiratory cycle. The onset and offset times of geniohyoid MU firing were related to the onset of each respiratory phase, and expressed both in seconds and as a percentage of the duration of the corresponding respiratory phase. The rate of MU firing was quantified both as the number of impulses per phase of the respiratory cycle, and as the mean rate of firing while active during both inspiration and expiration. The mean rate of firing while active was calculated from the number of impulses during each phase of the respiratory cycle and the duration of time over which these impulses occurred.

MU firing patterns and firing frequencies were averaged for 5-10 cycles during unoccluded breaths immediately preceding each occluded breath; the data associated with each occluded breath were averaged for each $\mathrm{MU}$. Subsequently means \pm SE were determined for the group of MU. Further statistical analyses included the paired $t$-test, the unpaired $t$-test, and linear regression using least-squares analysis. The criterion for statistical significance was $P<0.05$.

\section{Results}

Characteristics of geniohyoid MU. Recordings were obtained from 46 discriminated geniohyoid MU during unobstructed breathing in the 12 cats; several examples are shown in Fig. 1. Two subpopulations were identified; $23 \mathrm{MU}$ were active during inspiration only (I-MU) (e.g., Fig. 1, $A$ and $B$ ), and the other 23 were active during both inspiration and expiration (I/E-MU) (e.g., Fig. 1, $C$ and $D$ ). I-MU typically were active for only a portion of inspiration and usually became quiescent well before the end of inspiration. In contrast, I/E-MU generally fired throughout most of inspiration and continued to discharge during a substantial portion of expiration.

Frequency histograms of the relative onset and offset times of the geniohyoid MU are depicted in Fig. 2. Most of the I-MU started to fire between $5 \%$ and $20 \%$ of inspiratory time (Ti), although four I-MU had a later onset. In contrast, over half of the I/E-MU had an inspiratory onset of $<5 \%$ of inspiratory time, and all were active by $20 \%$ of inspiratory time. The mean relative inspiratory onset time was significantly earlier for the I/E-MU than for the I-MU $(5 \pm 1 \%$ Ti vs. $18 \pm 3 \% \mathrm{Ti} ; P$ $<0.002$ ). Once inspiratory activity had commenced, 21 of 23 I/E-MU were active for the remainder of inspiration, and in most cases I/E-MU firing continued without interruption into expiration. In contrast, 17 of 23 I-MU ceased firing well before the end of inspiration (the mean offset time for all 23 I-MU was $69 \pm 4 \% \mathrm{Ti}$ ) (Fig. 2). The result of the later onset and earlier offset times was that the mean duration of I-MU firing ( $52 \pm 5 \%$ $\mathrm{Ti}$ ) was significantly shorter than the mean duration of I/EMU inspiratory firing (92 $\pm 2 \% \mathrm{Ti}) ; P<0.0001$ ).

The expiratory activity of most I/E-MU started at expiratory onset and usually was an uninterrupted continuation of inspiratory activity (Figs. 1 and 2). For all but two I/E-MU, expiratory activity ceased before the end of expiration. Expiratory offset times were distributed broadly for I/E-MU (Fig. 2). The mean expiratory offset time for all I/E-MU was $73 \pm 3 \%$ of expiratory time (Te), and the mean duration of expiratory firing was $68 \pm 5 \% \mathrm{Te}$. The relative duration of expiratory firing was significantly shorter than the relative duration of inspiratory firing for the I/E-MU $(P<0.00001)$.

Fig. 3 depicts histograms for all geniohyoid MU of the number of impulses per breath and the mean MU firing frequency. The I-MU had a smaller number of spikes per inspiration than did the I/E-MU $(10 \pm 2$ vs. $16 \pm 2 ; P<0.02)$, as would 


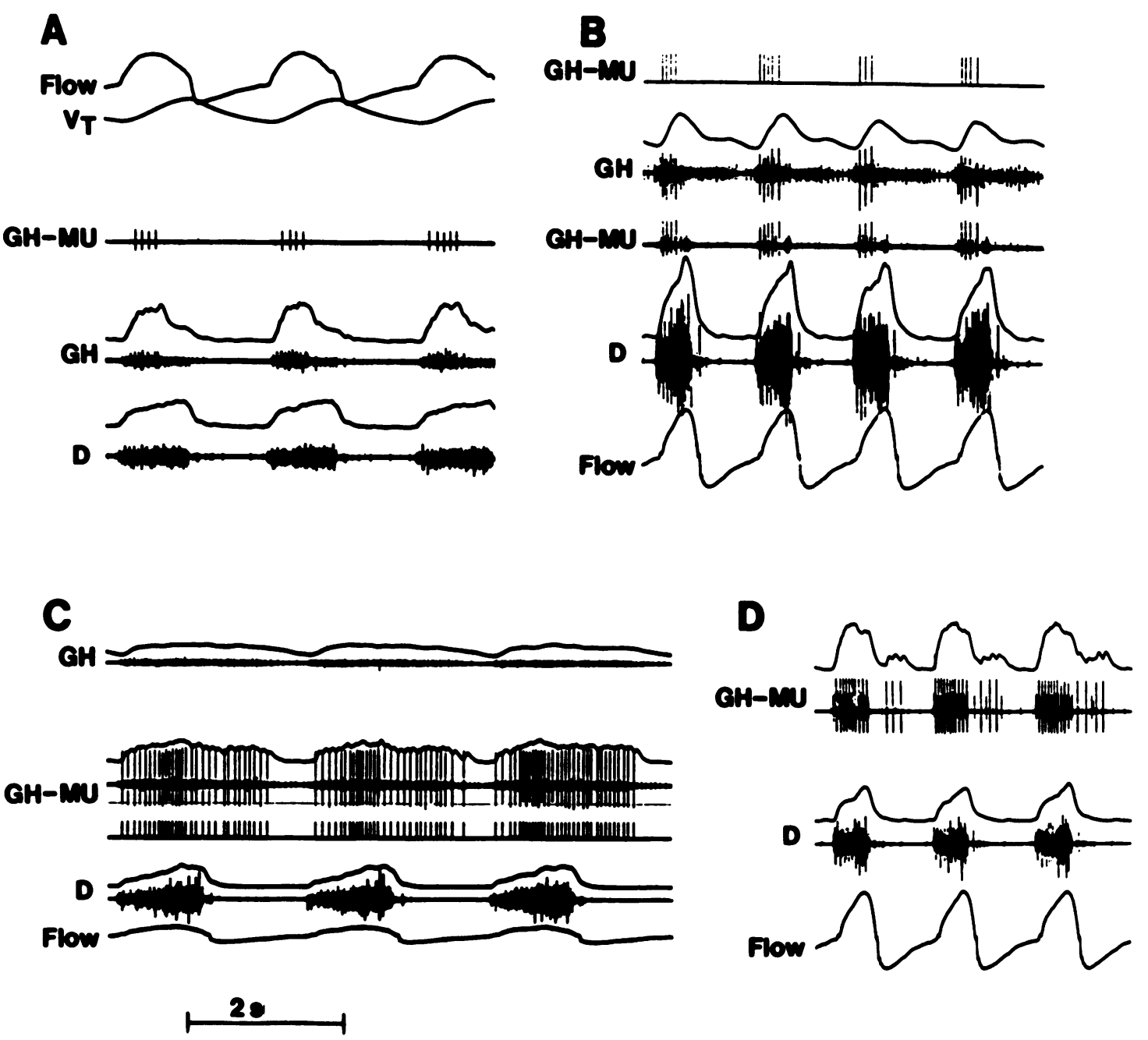

Figure 1. Examples of geniohyoid MU firing patterns during unobstructed breathing. ( $A$ and $B$ ) MU that fired during inspiration only (I-MU); $(C$ and $D)$ MU that were active during both inspiration and expiration (I/E-MU). Raw and moving average EMGs are depicted from the whole geniohyoid muscle $(G H)$, geniohyoid $\mathrm{MU}(G H-M U)$, and the diaphragm $(D)$. In $B$ and $C$, acceptance pulses from the window discriminator are also indicated as unipolar spikes which correspond to GH-MU activity. Flow and tidal volume $\left(V_{\mathrm{T}}\right)$ traces are depicted with upward deflections of the traces indicating inspiratory airflow and lung expansion, respectively. Time calibration, $2 \mathrm{~s}$.

be expected based on their shorter mean duration of inspiratory firing. The mean frequency of inspiratory firing was not significantly different for the two groups of MU, however. For the I/E-MU the number of impulses per inspiration was significantly greater than the number of impulses per expiration $(16 \pm 2$ vs. $10 \pm 1 ; P<0.0001)$, and furthermore, the mean frequency of inspiratory firing was significantly greater than the mean frequency of expiratory firing $(23.5 \pm 1.6$ vs. $15.7 \pm 1.3$ impulses per second; $P<0.00001$ ).

Effects of preventing lung expansion during inspiration. Occluding the airway at end-expiration prolonged the duration of the subsequent respiratory cycle, especially inspiratory time (from $0.74 \pm 0.04$ to $1.54 \pm 0.17 \mathrm{~s}$ ) but also expiratory time (from $0.93 \pm 0.04$ to $1.07 \pm 0.06 \mathrm{~s}$ ). Several examples of the effects of preventing lung expansion during inspiration on the firing patterns of geniohyoid MU are shown in Fig. 4. The absolute inspiratory onset times of the MU were not altered during end-expiratory occlusions. However, the absolute and relative durations of inspiratory firing were significantly prolonged for both the I-MU and the I/E-MU (Figs. 4 and 5). All
MU whose inspiratory activity continued until the end of inspiration during control breaths $(n=27)$ showed this same pattern of activity during end-expiratory occlusions. All MU whose inspiratory activity during control breaths terminated before the end of inspiration (17 I-MU and 2 I/E-MU) increased their relative time active during inspiration and delayed their relative inspiratory offset time (Fig. 6). Furthermore for these latter MU there were linear correlations between control and end-expiratory occluded breaths for relative time active $(r=0.763, P<0.001)$ and relative offset time $(r=0.484, P<0.05)$.

Preventing lung expansion during inspiration also augmented the frequency of geniohyoid MU firing (Fig. 5), on average increasing from $20.2 \pm 1.6$ to $25.8 \pm 2.0$ impulses per second for the I-MU and from $23.3 \pm 1.7$ to $27.4 \pm 2.2$ impulses per second for the I/E-MU. Increases in inspiratory firing frequency in response to end-expiratory airway occlusion occurred both during the time corresponding to the control portion of inspiration and during the time corresponding to the lengthened portion of inspiration (Fig. 7). As during control 

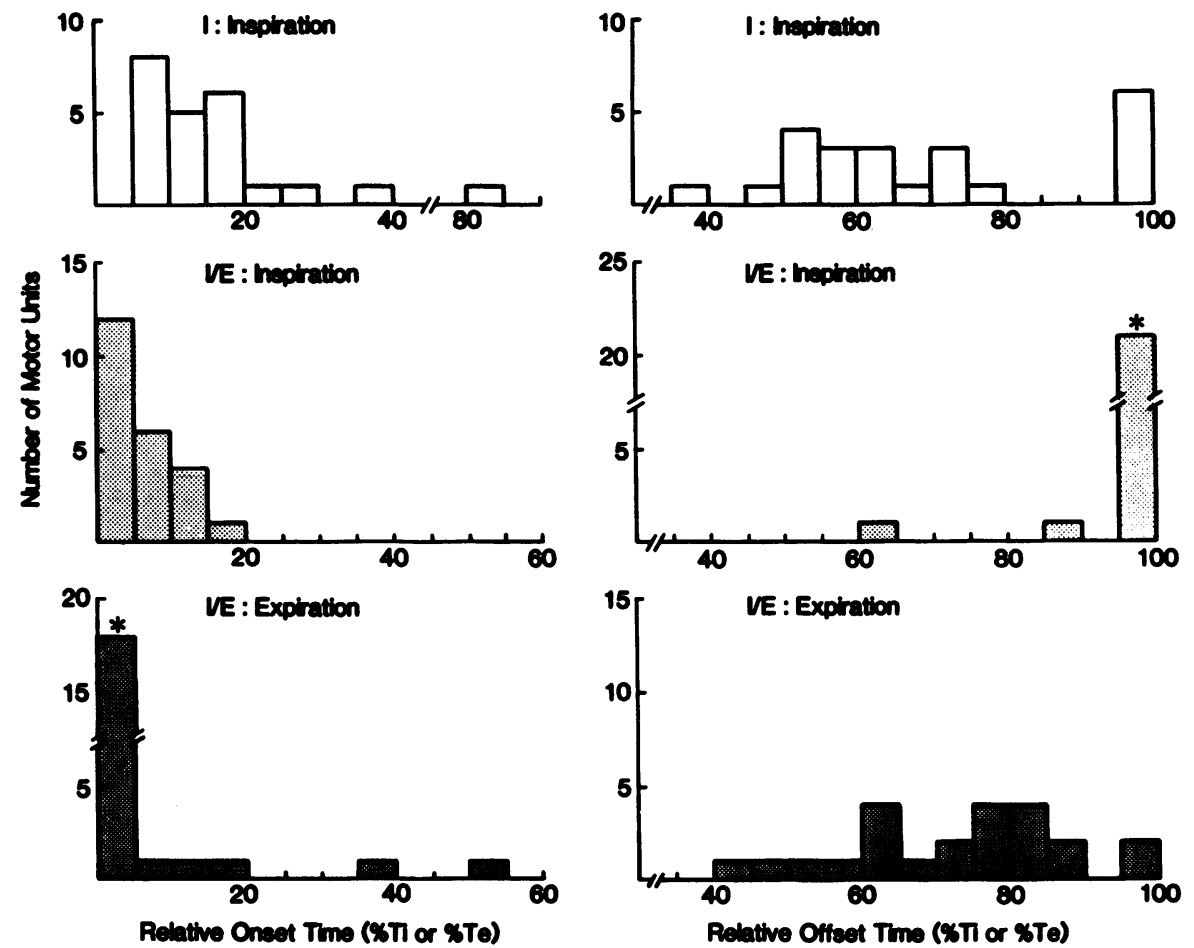

Figure 2. Frequency histograms of relative onset and offset times for geniohyoid MU during unobstructed breathing. Top panels depict data for MU that were active during inspiration only (I-MU). Middle and bottom panels depict inspiratory and expiratory data, respectively, for MU that were active during both inspiration and expiration (I/E-MU). ${ }^{*} 18$ of these MU remained active without a pause between the end of inspiration and the onset of expiration. $\mathrm{Ti}$, inspiratory time; $\mathrm{Te}$, expiratory time.

breaths, MU inspiratory firing frequency during end-expiratory occlusions was not significantly different for the I-MU compared to the I/E-MU. The increases in firing frequency and the prolongations of absolute duration of inspiratory activity during end-expiratory occluded breaths were appropri-
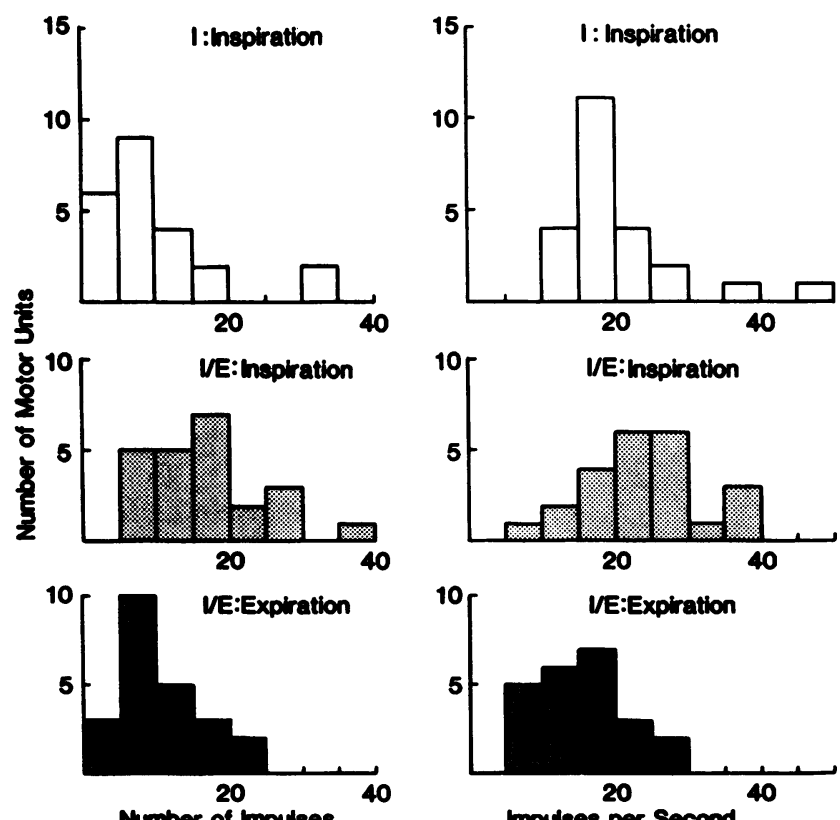

Figure 3. Frequency histograms for inspiratory and expiratory firing rates of geniohyoid MU during unobstructed breathing. Top panels depict data for MU that were active during inspiration only (I-MU). Middle and bottom panels depict inspiratory and expiratory data, respectively, for MU that were active during both inspiration and expiration (I/E-MU). ately reflected in significant augmentations in the total number of impulses per inspiration (Fig. 5).

The effects of preventing lung expansion during inspiration on geniohyoid MU firing during the subsequent expiration are shown in Figs. 4 and 8 . All 23 I-MU remained silent during expiration. Three of the I/E-MU became quiescent during the expiratory phase of end-expiratory occluded breaths, and the expiratory activity of most other I/E-MU was attenuated (Fig. 8). For the group of I/E-MU prevention of lung expansion during inspiration reduced the absolute firing period ( $P$ $<0.002)$, the relative firing period $(P<0.00002)$, the number of impulses per breath $(P<0.0001)$ and the mean frequency of firing $(P<0.001)$ during the subsequent expiration.

Effects of maintaining lung expansion during expiration. Recordings were obtained from 19 I-MU and 17 I/E-MU during end-inspiratory airway occlusions. On average expiratory time increased from $0.90 \pm 0.05$ to $2.73 \pm 0.35 \mathrm{~s}$. All I-MU remained silent during expiration when lung expansion was maintained (Fig. 9). The expiratory activity of I/E-MU was substantially stimulated by maintaining lung expansion during expiration (Figs. 9 and 10). Both the absolute and relative durations of MU firing were prolonged for all 17 I/E-MU, and for the group of MU these prolongations were statistically significant $(P<0.00002$ and $P<0.001$, respectively). The increase in the relative duration of activity resulted mainly from a delay in the relative offset time (from $72 \pm 4$ to $95 \pm 3 \%$ Te; $P$ $<0.0001$ ), as the expiratory onset times were altered only minimally. Maintaining lung expansion during expiration also significantly increased the number of impulses per expiration $(P<0.0001)$ and the mean frequency of expiratory firing $(P$ $<0.002$ ) of the geniohyoid I/E-MU (Fig. 10).

Maintenance of end-inspiratory lung expansion during the subsequent inspiration was performed while activity was recorded from nine I-MU and five I/E-MU (Fig. 9). Most of the MU were less active during inspiration at the elevated lung 

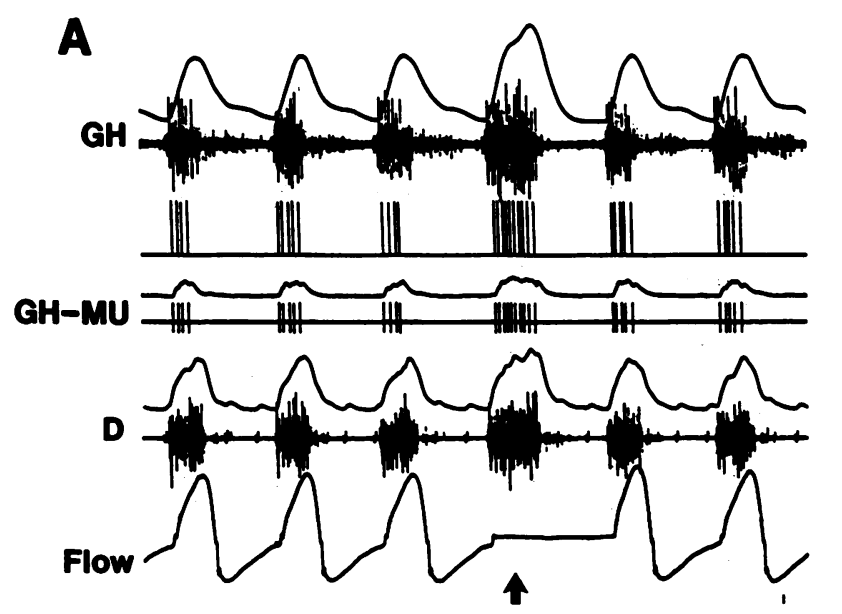

$\mathbf{B}$
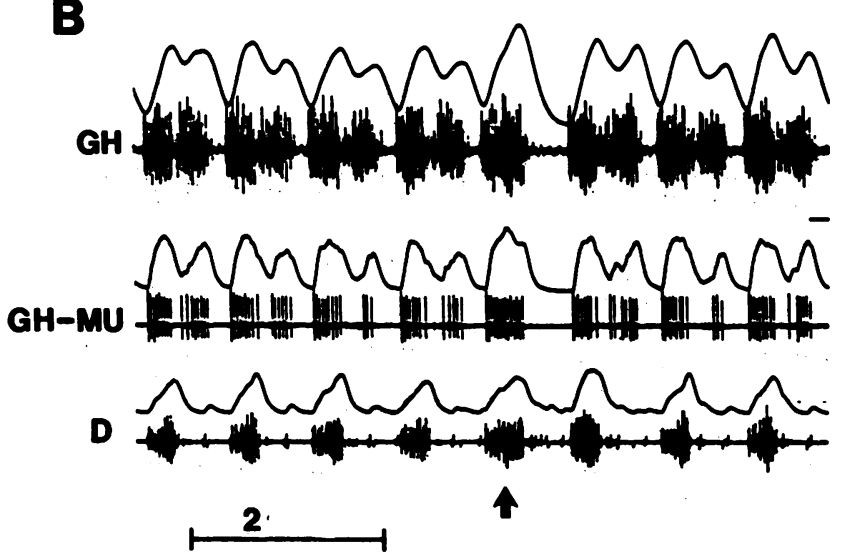

Figure 4. Examples of the effects of end-expiratory airway occlusion on firing patterns of geniohyoid MU $(G H-M U)$. $(A)$ MU that was active during inspiration only (I-MU); $(B)$ MU that was active during both inspiration and expiration (I/E-MU) of unobstructed breaths. Abbreviations and conventions are the same as in Fig. 1. The arrows depict the inspiratory phases of the respiratory cycles during which lung expansion was prevented.

volume, and six of the MU became silent (all of the latter were I-MU). For the group of $14 \mathrm{MU}$, the mean number of impulses per inspiration decreased from $14 \pm 2$ to $9 \pm 3(P<0.01)$ and the mean frequency of inspiratory firing slowed from $21.8 \pm 2.0$ to $10.6 \pm 3.0$ impulses per second $(P<0.0005)$.

\section{Discussion}

The present study examined the MU and hence the cellular basis for the neuromuscular control of pharyngeal patency. The findings indicate that the complex pattern of pharyngeal muscle activation results from the integrated activity of a heterogeneous population of $\mathrm{MU}$ with highly variable onset and offset times and firing frequencies, as opposed to a homogeneous population of MU with a single but complex pattern of activation. Changes in the activity of pharyngeal dilator muscles are the consequence of heterogeneous mechanisms, including MU recruitment and derecruitment, as well as alterations of the frequency and duration of firing of MU already
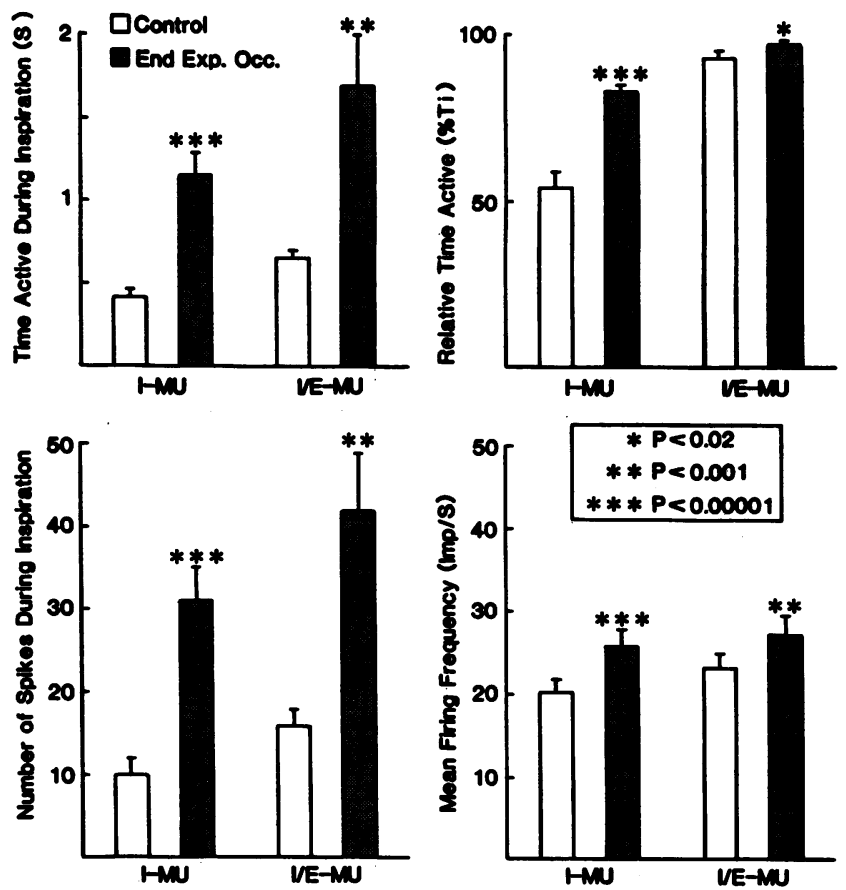

Figure 5. Mean effects ( \pm SE) of end-expiratory airway occlusion (End Exp. Occ.) on inspiratory activity of geniohyoid MU. Top panels indicate the absolute and relative durations of MU firing during inspiration, and bottom panels indicate MU firing frequencies expressed as number of spikes during inspiration and impulses per second $(\operatorname{Imp} / S)$ while active. Each panel depicts data for $23 \mathrm{MU}$ that fired only during inspiration of unoccluded breaths $(I-M U)$ and 23 MU that fired during both inspiration and expiration of unoccluded breaths $(I / E-M U)$. Significance of changes from control values is indicated by asterisks.

active. The results also confirm the inhibitory influence of volume-related afferents on upper airway dilating muscle activity during inspiration, and indicate a stimulatory influence on upper airway dilating muscle activity during expiration.

The geniohyoid muscle originates from the anterior aspect

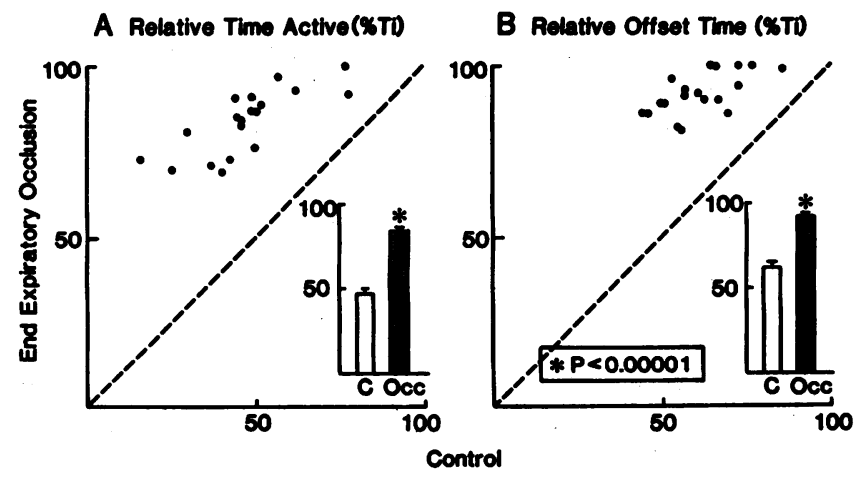

Figure 6. Effects of end-expiratory airway occlusion on the inspiratory activity of 19 geniohyoid MU (17 I-MU and 2 I/E-MU) which during unoccluded breaths had inspiratory offset times of $<100 \% \mathrm{Ti}$. Main panels indicate values for each of 19 MU (dashed line represents the line of identity), and insets indicate mean values ( $\pm \mathrm{SE})$ for all $19 \mathrm{MU}$ (asterisks indicate significance of changes from control). $C$, control; $O c c$, end-expiratory occlusion. 

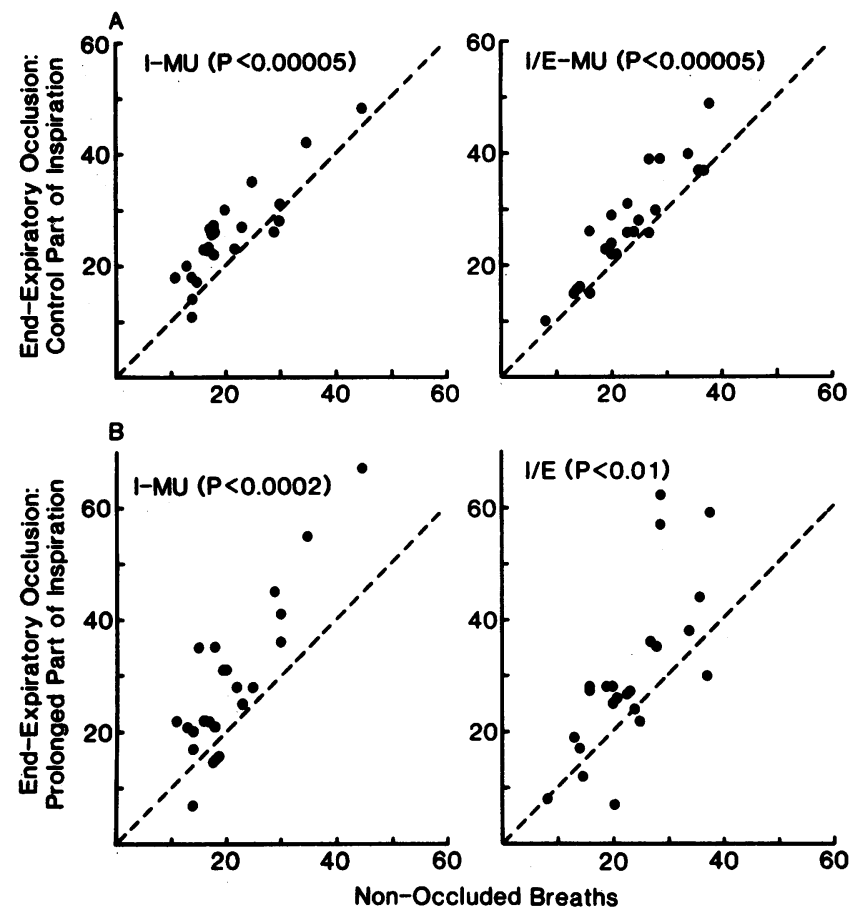

Figure 7. Effects of end-expiratory airway occlusion on MU inspiratory firing frequency during the time corresponding to the control portion of inspiration (top panels) and during the time corresponding to the lengthened portion of inspiration (bottom panels). Data for each MU during nonoccluded breaths ( $x$-axis) are compared to data during specific portions of occluded breaths ( $y$-axis); lines of identity are indicated. (Left panels) Data from I-MU; (right panels) data from I/E-MU. $P$ values in each panel indicate levels of significance for firing frequencies which were significantly higher during occluded compared to nonoccluded breaths.

of the mandible and inserts onto the freely movable hyoid arch $(9,34,35)$. Contraction of the geniohyoid dilates the pharyngeal airway and stabilizes the pharynx against collapsing forces
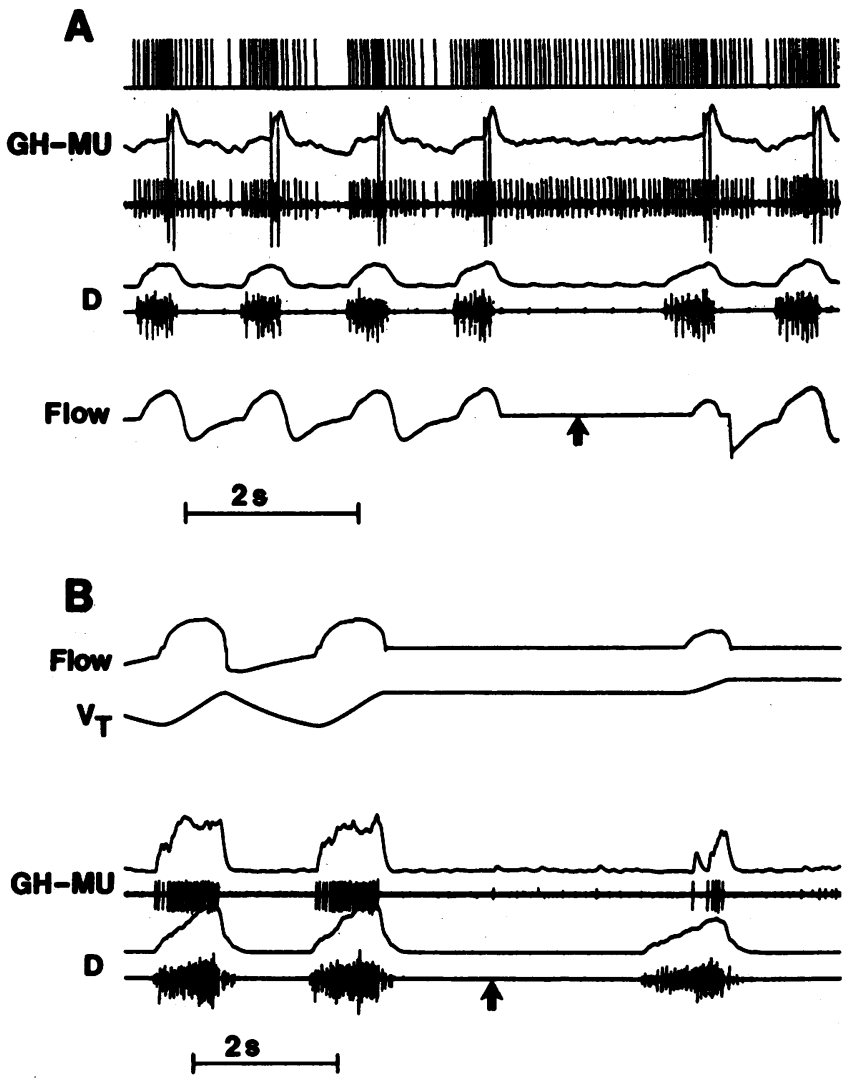

Figure 9. Examples of the effects of end-inspiratory airway occlusion on firing patterns of geniohyoid MU $(G H-M U)$. (A) Two MU, one of which was active during inspiration only (I-MU) and the other of which was active during both inspiration and expiration (I/E-MU). (B) MU that was active during inspiration (I-MU) of unobstructed breaths, and whose activity was substantially attenuated at an elevated lung volume. Abbreviations and conventions are identical to those of Fig. 1. The arrows depict the expiratory phase of the first respiratory cycle during which lung expansion was maintained.

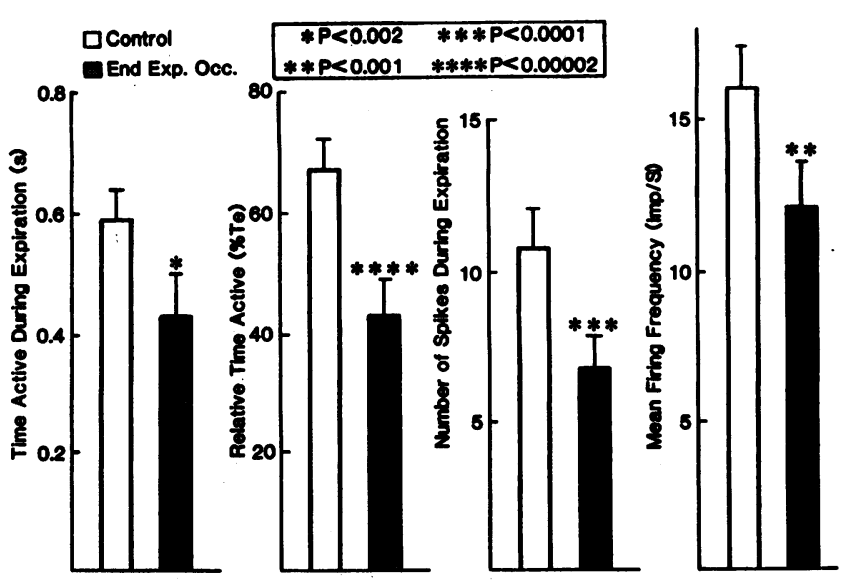

Figure 8. Mean effects $( \pm \mathrm{SE})$ of end-expiratory airway occlusion on the expiratory activity of the 23 geniohyoid MU which during unoccluded breaths were active during both inspiration and expiration (I/E-MU). (Left two panels) Absolute and relative durations of MU firing during expiration; (right two panels) MU firing frequencies expressed as number of spikes during expiration and impulses per second $(\operatorname{Imp} / S)$ while active. Significance of changes from control is indicated by asterisks.

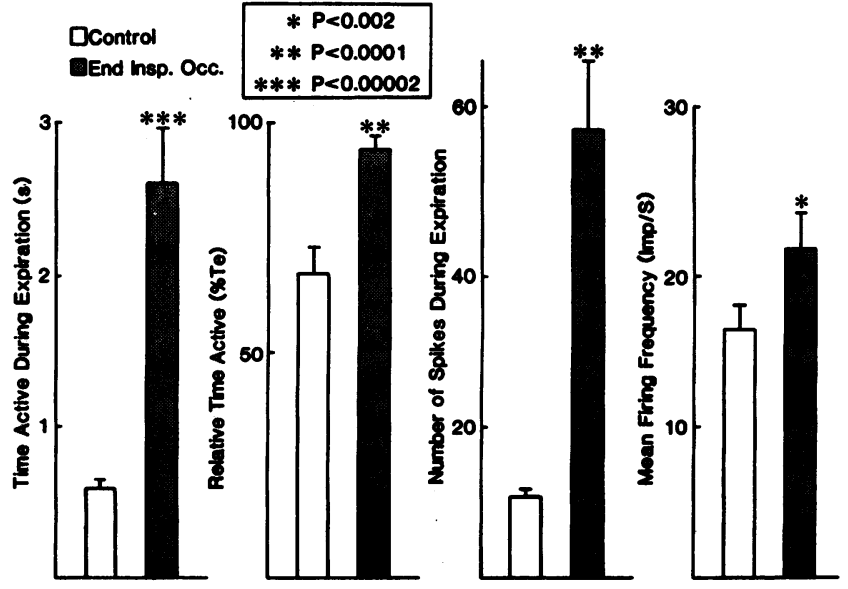

Figure 10. Mean effects ( \pm SE) of end-inspiratory airway occlusion (End Insp. Occ.) on the expiratory activity of 17 geniohyoid MU which during unoccluded breaths had been active during both inspiration and expiration (I/E-MU). (Left two panels) Absolute and relative durations of MU firing during expiration; (right two panels) MU firing frequencies expressed as number of spikes during expiration and impulses per second $(\operatorname{Imp} / S)$ while active. Significance of changes from control values are indicated by asterisks. 
resulting from subatmospheric pressures in the upper airways $(8,9,11,19,33)$. Located inferiorly to the genioglossus muscle, the geniohyoid muscle is a major contributor to the "submental" EMG recorded using surface electrodes in human subjects (37-39). The electrical activity of the geniohyoid muscle is phasically modulated during the respiratory cycle, and is influenced by many of the same chemical and mechanical stimuli which affect activity of the thoracic respiratory muscles (8, 9, 19). Furthermore, mechanical evidence suggests that the geniohyoid muscle may have a greater functional impact on respiratory movement of the hyoid arch than do certain infrahyoid muscles such as the sternohyoid muscle $(19,33)$.

Several differences were noted in the present study between geniohyoid I-MU and I/E-MU in the pattern of discharge during inspiration. The tendency for activity of I/E-MU to start firing earlier than the I-MU is reminiscent of feline phrenic nerve and diaphragm MU subpopulations, in which only the early onset MU continue to fire during the early part of expiration (24). However, in contrast to the diaphragm MU subpopulations, the geniohyoid I-MU and I/E-MU did not have clearly separable onset times. The generally earlier inspiratory offset times of I-MU compared to I/E-MU suggests that the former MU account for much of the mid- to late-inspiratory decline in the intensity of inspiratory activity typically noted in EMGs of upper airway dilating muscles, and the corresponding increase in upper airway resistance during late inspiration from its early- to mid-inspiratory nadir (12-20, 28, 40-42).

Neurophysiologically expiration has been divided into early and late phases $(24,36,43-46)$. The firing of geniohyoid I/E-MU during expiration did not appear to be strongly modulated by the two phases of expiration. Upper airway resistance increases during the course of expiration, reaching a peak value near end-expiration $(28,40-42)$. The smaller degree of pharyngeal dilator muscle activity during expiration compared to inspiration $(6-9,13-19,29)$ appears to result from many MU being silent (the I-MU) as well as reduced firing rates of MU which remain active (the I/E-MU). The wide distribution of expiratory offset times of geniohyoid I/E-MU suggests that an orderly quiescence of upper airway dilator MU accounts for the gradually incrementing airway resistance during the course of expiration. In two previous studies we noted that at times the geniohyoid muscle and the alae nasi muscle (a nasal dilator) underwent a period of shortening at or near mid-expiration $(19,35)$. MU were identified in the present study which can account for this behavior.

Preventing lung expansion during inspiration stimulates the peak and duration of upper airway dilating muscle EMG during inspiration, and in addition changes the early peaking pattern of electrical activity to one that is late-peaking $(12,17$, $29,30,32$ ). Consistent with the results of Sica et al. (12), we found that MU firing frequency increased, and, although not quantified in the present study, MU not active during unobstructed breathing were recruited in the absence of lung expansion. The effects of lung volume related afferents on upper airway muscle and cranial nerve activity during expiration are more controversial, with some studies indicating an augmentation and others a reduction in activity in the absence of lung inflation $(12,28,31,32)$. It was unclear from previous work whether regional differences might exist among airway dilating muscles in their expiratory responses to pulmonary stretch receptor afferents, or whether recordings from the hypoglossal nerve might be non-specific regarding pharyngeal dilator versus constrictor muscle activity. In the present study, in which MU activity was recorded from a well defined pharyngeal dilator $(8,9,11,15,33-35)$, lung volume-related afferents stimulated expiratory activity. Barillot and Dussardier (47) described laryngeal $M U$ which were tonically active but fired more rapidly during inspiration than during expiration. Lung volume afferents inhibited these MU during inspiration but excited them during expiration. This gating of afferent input during the respiratory cycle is consistent with the responses of the geniohyoid I/E-MU in the present study.

The influences of lung volume-related afferents on phrenic and diaphragm MU activity are inhibitory during both inspiration and expiration $(28,48)$. During inspiration, upper airway dilating muscles act synergistically with the diaphragm to maximize the rate and extent of lung expansion. During expiration, postinspiratory (early expiratory) activity of the diaphragm reduces the rate of expiratory airflow; in contrast, expiratory contraction of upper airway dilating muscles augments expiratory airflow by reducing upper airway resistance. Therefore, although lung volume-related afferents have opposite neurophysiological effects on the expiratory firing of the diaphragm and geniohyoid muscles, the reduction of diaphragm early expiratory activity and the augmentation of geniohyoid expiratory activity result in uniform mechanical consequences.

The present study was performed in acutely anesthetized as opposed to chronically instrumented nonanesthetized animals for several reasons: (a) Studies in anesthetized animals allow recordings to be made from a large number of $M U$, reducing the chance for random sampling error. (b) The quality of MU recordings is better in the anesthetized than in the chronically instrumented animal preparation. Lesser quality MU recordings have an inherent bias towards picking up well-discriminated activity from larger MU. MU of the phrenic and recurrent laryngeal nerves and of nerves supplying non-respiratory muscles are recruited sequentially on the basis of size $(25,47$, $49,50)$ and this is likely to be true for the hypoglossal nerve and geniohyoid muscle as well. Thus a bias towards recording from large $M U$ would substantially affect the analysis of $M U$ behavior. (c) Activity of the upper airway muscles is strongly influenced by relatively minor alterations in position of the head and neck (51), a problem which is avoided in an anesthetized animal model.

Respiratory-related activity of upper airway muscles and cranial nerves is influenced to a greater extent by anesthetics and sedatives than is the activity of the diaphragm and phrenic nerve (52-54). Similarly, slow wave and especially rapid eye movement sleep result in diminutions of upper airway muscle activity which may exceed reductions in diaphragm activity (55-57). It is likely that the sleep state and pharmacologic agents have different effects on MU activity. In the present study data were recorded under hypercapnic conditions to assess the behavior of MU with a wide variety of thresholds, and hence partially compensate for the depressive effects of anesthesia on MU activation. Nonetheless, the data of the present study may be more applicable to the patient with upper airway obstruction resulting from anesthetics and sedatives than to the patient with obstructive sleep apnea. Of interest, however, are reports indicating that agents such as alcohol and benzodiazepines which diminish upper airway muscle activity more than diaphragm activity $(52,58)$ may precipitate or worsen obstructive apneas during sleep (59-61). 


\section{Acknowledgments}

The authors thank Ronald Daniels for his excellent technical support. This research was supported in part by grants from the National Heart Lung and Blood Institute (HL-38701 and HL-25830). E. van Lunteren is the recipient of a Clinical Investigator Award from the National Heart Lung and Blood Institute (HL-01600).

\section{References}

1. Safar, P., L. A. Escarraga, and F. Chang. 1959. Upper airway obstruction in the unconscious patient. J. Appl. Physiol. 14:760-764.

2. Boidin, M. P. 1985. Airway patency in the unconscious patient. Br. J. Anesth. 57:306-310.

3. Plum, F., and J. B. Posner. 1980. The Diagnosis of Stupor and Coma. 3rd edition. F. A. Davis Co., Philadelphia. 347.

4. Adams, R., and M. Victor. 1981. Principles of Neurology. 2nd edition. McGraw-Hill Book Co., Inc., New York. 237.

5. Bannister, R. 1985. Brain's Clinical Neurology. 6th edition. Oxford University Press, London. 179.

6. Remmers, J. E., W. J. DeGroot, E. K. Sauerland, and A. M. Anch. 1978. Pathogenesis of airway occlusion during sleep. J. Appl. Physiol. 44:931-938.

7. Strohl, K. P., N. T. Feldman, N. A. Sanders, and M. Hallett. 1978. Obstructive sleep apnea in family members. N. Engl. J. Med. 299:969-973.

8. Brouillette, R. T., and B. T. Thach. 1979. A neuromuscular mechanism maintaining extrathoracic airway patency. J. Appl. Physiol. 46:772-779.

9. Van de Graaff, W. B., S. B. Gottfried, J. Mitra, E. van Lunteren, N. S. Cherniack, and K. P. Strohl. 1984. Respiratory function of the hyoid muscles and hyoid arch. J. Appl. Physiol. 57:197-204.

10. Roberts, J. L., W. R. Reed, and B. T. Thach. 1984. Pharyngeal airway-stabilizing function of sternohyoid and sternothyroid muscles in the rabbit. J. Appl. Physiol. 57:1790-1795.

11. Strohl, K. P., A. D. Wolin, E. van Lunteren, and J. M. Fouke. 1987. Assessment of muscle action on upper airway stability in anesthetized dogs. J. Lab. Clin. Med. 110:221-230.

12. Sica, A. L., M. I. Cohen, D. F. Donelly, and H. Zhang. 1984. Hypoglossal motoneuron responses to pulmonary and superior laryngeal afferent inputs. Respir. Physiol. 56:339-357.

13. Brouilette, R. T., and B. T. Thach. 1980. Control of genioglossus muscle inspiratory activity. J. Appl. Physiol. 49:801-808.

14. Mathew, O. P., Y. K. Abu-Osba, and B. T. Thach. 1982. Influence of upper airway negative pressure changes on genioglossus muscle respiratory activity. J. Appl. Physiol. 52:438-444.

15. Onal, E., M. Lopata, and T. D. O'Connor. 1981. Diaphragmatic and genioglossal electromyogram responses to isocapnic hypoxia in humans. Am. Rev. Respir. Dis. 124:215-217.

16. Haxhiu, M. A., E. van Lunteren, J. Mitra, and N. S. Cherniack. 1987. Comparison of the response of diaphragm and upper airway dilating muscle activity in sleeping cats. Respir. Physiol. 70:183-193.

17. van Lunteren, E., K. P. Strohl, D. M. Parker, E. N. Bruce, W. B. Van de Graaff, and N. S. Cherniack. 1984. Phasic volume-related feedback on upper airway muscle activity. J. Appl. Physiol. 56:731736.

18. van Lunteren, E., W. B. Van de Graaff, D. M. Parker, J. Mitra, M. A. Haxhiu, K. P. Strohl, and N. S. Cherniack. 1984. Nasal and laryngeal reflex responses to negative upper airway pressure. J. Appl. Physiol. 56:746-752.

19. van Lunteren, E., M. A. Haxhiu, and N. S. Cherniack. 1987. Mechanical function of hyoid muscles during spontaneous breathing in cats. J. Appl. Physiol. 62:582-590.

20. Weiner, D., J. Mitra, J. Salamone, and N. S. Cherniack. 1982. Effect of chemical stimuli on nerves supplying upper airway muscles. J. Appl. Physiol. 52:530-536.

21. Buchtal, F., and H. Schmalbruch. 1980. Motor unit of mammalian muscle. Physiol. Rev. 60:90-142.
22. St. John, W. M., and D. Bartlett, Jr. 1979. Comparison of phrenic motoneuron responses to hypercapnia and isocapnic hypoxia. J. Appl. Physiol. 46:1096-1102.

23. Donnelly, D. F., M. I. Cohen, A. L. Sica, and H. Zhang. 1985. Responses of early and late onset phrenic motoneurons to lung inflation. Respir. Physiol. 61:69-83.

24. Prabhakar, N. P., J. Mitra, J. L. Overholt, and N. S. Cherniack. 1986. Analysis of postinspiratory activity of phrenic motoneurons with chemical and vagal reflexes. J. Appl. Physiol. 61:1499-1509.

25. Dick, T. E., F. J. Kong, and A. J. Berger. 1987. Correlation of recruitment order with axonal conduction velocity for supraspinally driven diaphragmatic motor units. J. Neurophysiol. 57:245-259.

26. Hwang, J.-C., D. Bartlett, Jr., and W. M. St. John. 1983. Characterization of respiratory-modulated activities of hypoglossal motoneurons. J. Appl. Physiol. 55:793-798.

27. Mitra, J., and N. S. Cherniack. 1983. The effects of hypercapnia and hypoxia on single hypoglossal nerve fiber activity. Respir. Physiol. 54:55-66.

28. Bartlett, D., Jr., J. E. Remmers, and H. Gautier. 1973. Laryngeal regulation of respiratory airflow. Respir. Physiol. 18:194-204.

29. Kuna, S. T. 1987. Interaction of hypercapnia and phasic volume feedback on motor control of the upper airway. J. Appl. Physiol. 63:1744-1749.

30. Cohen, M. I. 1975. Phrenic and recurrent laryngeal discharge patterns and the Hering-Breuer reflex. Am. J. Physiol. 228:1489-1496.

31. van Lunteren, E., M. A. Haxhiu, and N. S. Cherniack. 1985. Respiratory changes in nasal muscle length. J. Appl. Physiol. 59:453458.

32. Sica, A. J., M. I. Cohen, D. F. Donnelly, and H. Zhang. 1985. Responses of recurrent laryngeal motoneurons to changes of pulmonary afferent inputs. Respir. Physiol. 62:153-168.

33. van Lunteren, E., M. A. Haxhiu, and N. S. Cherniack. 1987. Relation between upper airway volume and hyoid muscle length. $J$. Appl. Physiol. 63:1443-1449.

34. DeBrul, E. L. 1980. Sicher's Oral Anatomy. 7th edition. C. V. Mosby Co., St. Louis, MO. 336-350.

35. Crouch, J. E. 1969. Text-Atlas of Cat Anatomy. Lea and Febiger, Philadelphia.

36. van Lunteren, E., N. S. Cherniack, and T. E. Dick. 1988. Upper airway pressure receptors alter expiratory muscle EMG and motor unit firing. J. Appl. Physiol. 65:210-217.

37. Jeffries, B., R. T. Brouilette, and C. E. Hunt. 1984. Electromyographic study of some accessory muscles of respiration in children with obstructive sleep apnea. Am. Rev. Respir. Dis. 129:696-702.

38. Hudgel, D. W., K. R. Chapman, C. Faulks, and C. Hendricks. 1987. Changes in inspiratory muscle electrical activity and upper airway resistance during periodic breathing induced by hypoxia during sleep. Am. Rev. Respir. Dis. 135:899-906.

39. Suratt, P. M., R. F. McTier, and S. C. Wilhoit. 1988. Upper airway muscle activation is augmented in patients with obstructive sleep apnea compared with that in normal subjects. Am. Rev. Respir. Dis. 137:889-894.

40. Orem, J., A. Netick, and W. C. Dement. 1977. Increased upper airway resistance to breathing during sleep in the cat. Electroencephalogr. Clin. Neurophysiol. 43:14-22.

41. Bartlett, D. 1979. Effects of hypercapnia and hypoxia on laryngeal resistance to airflow. Respir. Physiol. 37:293-302.

42. Sanders, M. H., and S. E. Moore. 1983. Inspiratory and expiratory partitioning of airway resistance during sleep in patients with sleep apnea. Am. Rev. Respir. Dis. 127:554-558.

43. Ballantyne, D., and D. W. Richter. 1986. The non-uniform character of expiratory synaptic activity in expiratory bulbospinal neurones of the cat. J. Physiol. (Lond.). 370:433-456.

44. Remmers, J. E., D. W. Richter, D. Ballantyne, C. R. Bainton, and J. P. Klein. 1986. Reflex prolongation of stage I of expiration. Pflügers Arch. Eur. J. Physiol. 407:190-198.

45. Ledlie, J. F., A. I. Pack, and A. P. Fishman. 1983. Effects of 
hypercapnia on abdominal nerve expiratory activity. J. Appl. Physiol. 55:1614-1622.

46. DeTroyer, A., and V. Ninane. 1986. Triangularis sterni: a primary muscle of breathing in the dog. J. Appl. Physiol. 60:14-21.

47. Barillot, J. C., and M. Dussardier. 1973. Modalités de décharge des motoneurones laryngés inspiratoires dans diverses conditions expérimentales. J. Physiol. (Paris). 66:593-629.

48. Hilaire, G., and R. Monteau. 1979. Facteurs déterminant l'ordre de recrutement des motoneurones phréniques. J. Physiol. (Paris). 75:765-781.

49. Henneman, E., G. Somjen, and D. O. Carpenter. 1965. Functional significance of cell size in spinal motoneurons. J. Neurophysiol. 28:560-580.

50. Henneman, E., G. Somjen, and D. O. Carpenter. 1965. Excitability and inhibitibility of motoneurons of different sizes. J. Neurophysiol. 28:599-620.

51. Bonora, M., D. Bartlett, Jr., and S. L. Knuth. 1985. Changes in upper airway muscle activity related to head position in awake cats. Respir. Physiol. 60:181-192.

52. Bonora, M., W. M. St. John, and T. A. Bledsoe. 1985. Differential elevation by protriptyline and depression by diazepam of upper airway respiratory motor activity. Am. Rev. Respir. Dis. 131:41-45.

53. Bruce, E. N., J. Mitra, and N. S. Cherniack. 1982. Central and peripheral chemoreceptor inputs to phrenic and hypoglossal motorneurons. J. Appl. Physiol. 53:1504-1511.

54. Hwang, J. C., W. M. St. John, and D. Bartlett, Jr. 1983. Respiratory-related hypoglossal nerve activity: influence of anesthetics. $J$. Appl. Physiol. 55:785-792.

55. Sauerland, E. K., and R. M. Harper. 1976. The human tongue during sleep: electromyographic activity of the genioglossus muscle. Exp. Neurol. 51:160-170.

56. Orem, J., P. Norris, and R. Lydic. 1978. Laryngeal abductor activity during sleep. Chest. 73(Suppl.):300-301.

57. Parisi, R. A., T. V. Santiago, and N. H. Edelman. 1988. Genioglossal and diaphragmatic EMG responses to hypoxia during sleep. Am. Rev. Respir. Dis. 138:610-616.

58. Krol, R. C., S. L. Knuth, and D. Bartlett, Jr. 1984. Selective reduction of genioglossal muscle activity by alcohol in normal human subjects. Am. Rev. Respir. Dis. 129:247-250.

59. Taasan, V. C., A. J. Block, P. G. Boysen, J. W. Wynne, C. White, and S. Lindsey. 1981. Alcohol increases sleep apnea and oxygen desaturation in asymptomatic men. Am. J. Med. 71:240-245.

60. Issa, F. G., and C. E. Sullivan. 1982. Alcohol, snoring and sleep apnea. J. Neurol. Neurosurg. Psychiatry. 45:353-359.

61. Dolly, F. R., and A. J. Block. 1982. Effect of flurazepam in sleep-disordered breathing and nocturnal oxygen desaturation in asymptomatic subjects. Am. J. Med. 73:239-243. 